\title{
Anpassungs- und Gestaltungsfähigkeit regionaler Systeme beruflicher Weiterbildung
}

\author{
Eine institutionenökonomische Analyse
}

\author{
Anne Margarian (D) Matthias Lankau
}

Eingegangen: 18. Oktober 2017 / Angenommen: 27. Februar 2018 / Online publiziert: 12. März 2018

(C) Der/die Autor(en) 2018. Dieser Artikel ist eine Open-Access-Publikation.

Zusammenfassung Berufliche Weiterbildung (bW) wird als wichtiges Element moderner Wissensökonomien betrachtet. Basierend auf Experteninterviews in vier Fallstudienregionen analysiert die Studie Mechanismen der Koordinierung von Nachfrage und Angebot, die Strategien der Anbieter und die Leistung der regionalen Systeme der bW. Die zunehmende Modularisierung und Digitalisierung ermöglicht auch in weniger attraktiven peripheren Märkten Angebote der bW, erschwert aber eine Ko-Produktion von Wissen, die gerade hier für eine erhöhte Beteiligung Beschäftigter an bW Voraussetzung wäre.

Schlüsselwörter Berufliche Weiterbildung · Hierarchien · Ko-Produktion von Wissen $\cdot$ Netzwerksteuerung $\cdot$ Regionalentwicklung

\section{Adaptability and creative potential of local further training systems}

Assessments from an institutional economics perspective

\begin{abstract}
Further training (FT) is an important element of the modern knowledge economy. Based on expert interviews from four case-study regions, the paper analyses the relevance of markets, hierarchies, and networks as coordination mechanisms in FT, the strategies of providers, and the resulting performance of local FT service systems. We find increasing modularisation and digitalisation of FT services, which
\end{abstract}

\footnotetext{
Publisher's Note Springer Nature remains neutral with regard to jurisdictional claims in published maps and institutional affiliations.

Dr. A. Margarian $(\bowtie)$

Thünen-Institut für Ländliche Räume, Bundesallee 50, 38116 Braunschweig, Deutschland

E-Mail: anne.margarian@thuenen.de

Dr. M. Lankau

Handwerkskammer Hannover, Berliner Allee 17, 30175 Hannover, Deutschland

E-Mail: lankau@hwk-hannover.de
} 
enables FT provision in less attractive markets but does not contribute to the value coproduction required for an increase of FT participation of employees.

Keywords Further training $\cdot$ Hierarchies $\cdot$ Knowledge co-production $\cdot$ Networkgovernance $\cdot$ Regional development

\section{Einleitung}

Die Entwicklung westlicher Ökonomien hin zu wissensbasierten Wirtschaften hat die Erkenntnis mit sich gebracht, dass Erwachsenenbildung und ,Lebenslanges Lernen“ wichtige Bedingungen für die soziale und wirtschaftliche Entwicklung sind (Borrás und Edquist 2015). Die berufliche Weiterbildung (bW) dient dem Erwerb von Fertigkeiten und Kenntnissen, die im Beschäftigungssystem (Brater 1980), und damit vor allem in den Unternehmen, benötigt werden. Sie wird als bedeutsam für funktionierende Arbeitsmärkte, für die konstante Weiterentwicklung der beruflichen Fähigkeiten der arbeitsfähigen Bevölkerung sowie für die Innovationsfähigkeit der Wirtschaft angesehen (Bosch und Charest 2008).

Aus der ökonomischen, marktzentrierten Perspektive ist die Koordination zwischen Angebot und Nachfrage der bW durch zwei Kernprobleme charakterisiert: Erstens ist aufgrund positiver externer Effekte effektiver bW ihr gesellschaftlicher Nutzen größer als die Summe der privaten Nutzen, so dass das Weiterbildungsengagement, wenn es alleine privater Initiative überlassen bleibt, aus gesellschaftlicher Sicht zu gering ausfällt. Zweitens müssen Angebote zur beruflichen Weiterbildung unter der Restriktion unvollkommener Information entwickelt werden. Bei der beruflichen Weiterbildung handelt es sich um eine wissensintensive Dienstleistung, deren Form und Inhalt aufgrund sich wandelnder Managementstrategien und technologischer Entwicklungen konstant an neue Anforderungen angepasst werden müssen, so dass eine intensive Zusammenarbeit zwischen Anbieter und Kunden nötig ist (Tietgens 1991). Die Dienstleistungsforschung hat diese Erfordernisse unter der Idee der gemeinsamen Erzeugung von Dienstleistungen und Werten (,service co-production“ und „,value co-creation“; Lusch und Vargo 2006) erfasst, für die spezifische Konfigurationen von Akteuren und geteilter Information erforderlich sind (Maglio und Spohrer 2008).

In der bW kommt erschwerend hinzu, dass sowohl Beschäftigte und Arbeitssuchende als auch Arbeitgeber als Kunden von Weiterbildungsanbietern auftreten. Informationen und Bedarfe dieser Kundengruppen können voneinander abweichen. Arbeitgeber organisieren, oft in Zusammenarbeit mit externen Anbietern, betriebliche Weiterbildung, die insbesondere bei großen Unternehmen neben bW auch allgemeine kulturelle oder politische Bildungsangebote mit einschließen kann (Nuissl und Brandt 2009). Arbeitsmarktpolitische Weiterbildung hingegen fördert meist die Teilnahme Arbeitssuchender und manchmal auch Beschäftigter an Angeboten zur bW. Darüber hinaus können Arbeitssuchende und Beschäftigte, unabhängig von Arbeitgebern und arbeitsmarktpolitischer Förderung, privat finanziert an entsprechenden Angeboten zur bW außerhalb betrieblicher Kontexte teilnehmen. Viele Weiterbildungsanbieter adressieren betriebliche, arbeitsmarktpolitische und individuelle 
berufliche Weiterbildung gleichermaßen, um positive Größen- und Synergieeffekte realisieren zu können. Damit sie das Ziel, die Beschäftigungs- und Beförderungswürdigkeit ihrer individuellen Kunden zu erhöhen, realisieren können, müssen sie die Bedarfe der Unternehmen an Fähigkeiten und Kenntnissen möglichst genau kennen.

Aufgrund der Besonderheiten des Marktes für bW und seiner gesellschaftlichen Bedeutung greift der Staat an verschiedenen Stellen steuernd darin ein. Weil aber im Bereich der bW nicht nur Märkte sondern auch die Politik als alleinige Koordinationsmechanismen aufgrund grundsätzlicher Probleme zu scheitern drohen (Robalino et al. 2012), sind differenziertere Koordinationsmechanismen für die Sicherung eines aus gesellschaftlicher Sicht optimalen Angebots bzw. einer optimalen Nachfrage an bW wichtig. Auf nationaler Ebene wurden die entsprechenden Institutionen der Koordination bereits vielfach untersucht (z. B. Bosch 2017). Die Unterschiede der Ausund Weiterbildungssysteme verschiedener Länder sind zum Beispiel ein wichtiges Thema der ,varieties of capitalism“-Literatur. Finegold und Soskice haben 1988 mögliche Koordinationsprobleme bestimmter institutioneller Systeme der Aus- und Weiterbildung identifiziert und gezeigt, dass diese zu einem ,high-skill“-Gleichgewicht in einer Nation und einem ,low-skill“-Gleichgewicht in anderen Nationen führen könnten (Finegold und Soskice 1988). Ein Jahrzehnt später unterzog Finegold (1999) seinen eigenen Ansatz einer kritischen Überprüfung. Er kam zu dem Schluss, dass die Unterscheidung in zwei klar getrennte Gleichgewichte und der statische Charakter des Gleichgewichtsmodells der Vielfalt der empirischen Befunde nicht gerecht werden konnte und entwarf die Idee der ,skill-ecosystems“. Buchanan (2006, S. 14) wendete das Konzept im australischen Kontext an und definierte „skill-ecosystems“ als „Cluster unterschiedlicher Kompetenzen in einer spezifischen Region oder Branche, die durch verflochtene Netzwerke aus Unternehmen, Märkten und Institutionen geprägt werden“.

Obwohl es erhebliche Unterschiede in der Weiterbildungsbeteiligung zwischen deutschen Landkreisen gibt (Martin und Schrader 2016), wurden die entsprechenden Koordinationsmechanismen für Angebot und Nachfrage von Weiterbildungsangeboten auf der sub-nationalen Ebene Deutschlands bisher nur selten analysiert. Systematisch untersucht wurden bisher vor allem Einflüsse der betrieblichen Umgebung auf die Beteiligung an bW (Martin et al. 2015). Martin und Schömann (2015) haben die Bedeutung der Steuerung auf kommunaler Ebene vor dem Hintergrund der Umstellung auf Methoden des „New Public Management“ in der Arbeitsverwaltung und die Diskussion der sich wandelnden Rolle des Staates im Kontext des „Governance“-Konzeptes untersucht und herausgefunden, dass der kommunalen Ebene in der Erklärung der Weiterbildungsbeteiligung eine höhere Bedeutung zukommt als den größeren Raumeinheiten. Zu den wenigen entsprechenden Studien gehört auch Reginis (1997) Untersuchung der Anpassungsstrategien von Unternehmen und Institutionen an neue Anforderungen an die Aus- und Weiterbildung in vier europäischen Fallregionen. Seine Analyse zeigt, dass der Umgang mit den oft regionsspezifischen Herausforderungen ebenso regionsspezifische Antworten erfordert, so dass die Strategien der Regionen sich auch unabhängig von Unterschieden im nationalen institutionellen Überbau unterscheiden. 
Vor diesem Hintergrund fragt die vorliegende Untersuchung, durch welche Mechanismen und in welchem Ausmaß Bedarfe und Angebote der bW in regionalen Weiterbildungssystemen aufeinander abgestimmt werden. Die Arbeit geht davon aus, dass regionale Unterschiede in Markt- und Netzwerkstrukturen sich in unterschiedlichen Managementstrukturen, Strategien und Leistungen niederschlagen. In der Analyse wird ein institutionenökonomisch fundierter konzeptioneller Rahmen erprobt, mithilfe dessen die Koordinationsfähigkeit regionaler Systeme der beruflichen Weiterbildung untersucht und bewertet werden kann. Die Daten für die Untersuchung wurden im Rahmen von explorativen regionalen Fallstudien in Experteninterviews mit Beteiligten im System der bW erhoben.

Der Artikel beginnt im folgenden Kap. 2 mit der Zusammenfassung der Literatur zu den Koordinationsstrukturen Hierarchie, Markt und Netzwerk im Bereich der bW. Kap. 3 erläutert den Analyserahmen sowie Kontext und die Herangehensweise der empirischen Untersuchung in den vier Fallregionen. Es präsentiert dann die Ergebnisse zur Steuerung über lokale Märkte, Hierarchien und Netzwerke sowie zu Strategien der Beteiligten und Leistungen der regionalen Systeme. Kap. 4 zieht Schlussfolgerungen mit Blick auf die politische Zielsetzung, eine verstärkte Weiterbildungsbeteiligung und regionale Gleichwertigkeit zu erreichen. Es bemüht sich außerdem um eine Beurteilung der Fruchtbarkeit des angewendeten heuristischen Untersuchungskonzeptes für die Analyse.

\section{Strukturen des deutschen Systems der beruflichen Weiterbildung}

Wegge (1996) hat auf den Zusammenhang zwischen den besonderen Gütereigenschaften der Weiterbildung als private oder öffentliche Dienstleistung und dem Erfolg unterschiedlicher institutioneller Arrangements hingewiesen. Die folgende Beschreibung der spezifischen Struktur des deutschen Weiterbildungssystems anhand der existierenden Literatur folgt der Einteilung der Koordinationsmechanismen von Powell (1990) in Hierarchien, Märkte und Netzwerke.

\subsection{Hierarchien in der bW}

Auch wenn in Bezug auf bW ab und zu ein (staatliches) Steuerungsdefizit beklagt wird, ist die bW in Deutschland doch nach wie vor stark durch staatliche Organisationen, namentlich vor allem die Agentur für Arbeit, und ihr Handeln geprägt (Hartz und Schrader 2008). Einen gesetzlichen Rahmen bilden auf der Bundesebene vor allem das Berufsbildungsgesetz (BBiG) sowie das zweite und dritte Sozialgesetzbuch (SGB II und SGB III), die die Förderung von Arbeitslosen bzw. Langzeitarbeitslosen regulieren (weiterführend vgl. z. B. Grotlüschen et al. 2010; oder Nuissl und Brandt 2009). SGB III und SGB II werden durch die Bundesagentur für Arbeit mit den Agenturen für Arbeit (AfA) bzw. durch die Jobcenter in den Regionen umgesetzt. Die AfA vor Ort erstellen in der Regel jedes Jahr Bildungszielpläne, in denen die Erwartungen der Bedarfe der Agentur im Bereich der Weiterbildung im kommenden Jahr beschrieben werden, und an denen sich die Bildungsanbieter in der Planung ihrer Kurse orientieren können (Doerr und Kruppe 2012). 
In den letzten Jahrzenten wurde versucht, die Handlungsspielräume der Angestellten sowie Beamten und Beamtinnen der Arbeitsverwaltung durch eher marktähnliche Anreizsysteme und Managementpraktiken des „New Public Management“ (Osborne und Plastrik 1999) zu erhöhen. So wurde in den AfA die Vorschrift bestimmter Routinen zunehmend durch die Setzung bestimmter Ziele und Zielwerte ersetzt, das heißt statt der Mittel und Wege werden jetzt vor allem die Ergebniserwartungen definiert (Sowa et al. 2016). Dementsprechend können sich einzelne Jobcenter und AfA auch im Bereich der Förderung bW in ihren Strategien voneinander unterscheiden (vgl. z. B. Klingert und Lenhart 2017; Sowa et al. 2016; Dauth 2017). Strategieunterschiede bestehen auch zwischen Jobcentern, die als sogenannte „Optionskommunen“ direkt in die Kommunalverwaltung integriert sind, und Jobcentern im Geschäftsbereich der Bundesagentur für Arbeit (Margarian und Lilje 2014). Der Erfolg von Mitarbeitenden und Dienststellen der Arbeitsverwaltung wird vor allem am durch entsprechende Indikatoren dokumentierten Erfolg der Integration in Arbeit gemessen. Im Ergebnis kann es durch die Zielsteuerung zu größeren Freiheitsgraden der Mitarbeiter und Mitarbeiterinnen kommen, die sich dann möglicherweise besser in regionale Netzwerke einbringen und Flexibilität im Umgang mit lokal spezifischen Ansprüchen gewinnen können. Andererseits sind inzwischen auch die möglichen problematischen Aspekte einer Steuerung über zu einseitige Zielindikatoren deutlich geworden (Sowa und Staples 2014; Schütz 2008), wie zum Beispiel eine mögliche selektive Förderung von Fällen mit hoher Erfolgswahrscheinlichkeit.

Auf der Angebotsseite gibt es private und öffentliche sowie gewinnorientierte und nicht-gewinnorientierte Anbieter. Als öffentliche oder quasi-öffentliche Einrichtungen mit hoheitlichem oder öffentlichem Auftrag sind die Industrie- und Handelskammern (IHK), Handwerkskammern (HWK) sowie die Volkshochschulen (VHS) tätig. Die Kammern haben durch ihren hoheitlichen Auftrag im Bereich der Ausund Weiterbildung eine Sonderstellung untern den Anbietern. Die VHS bedienen das Segment der bW je nach eigener Strategie und regionalen Gegebenheiten in sehr unterschiedlichem Maße; sie haben den öffentlichen Auftrag, Bildung in die Fläche zu tragen, und erhalten zu seiner Erfüllung eine staatliche Grundfinanzierung. Kirchliche Einrichtungen bieten oft bW im sozialen Bereich an. Aufstiegsweiterbildung gibt es auch an Berufsschulen, Fachhochschulen und Technikerschulen.

Auf Seiten der privaten gewinnorientierten und gemeinnützigen Anbieter wird der deutsche Markt von großen Organisationen mit komplexen Organisationsstrukturen und mehreren Hierarchieebenen bestimmt, unter ihnen gewerkschafts- und arbeitgebernahe Institutionen. Die jeweilige Organisationsstruktur prägt Strategie und Verhalten von Mitarbeitenden und Management gewinn- und nicht-gewinnorientierter Weiterbildungsanbieter gleichermaßen, wie Zech (2013) zeigen konnte.

\subsection{Märkte in der bW}

Büchter und Gramlinger haben 2005 festgestellt, dass das deutsche System der beruflichen Weiterbildung trotz der steuernden Eingriffe des Staates (s. Abschn. 2.1) im Vergleich zu anderen europäischen Systemen als marktbasiert bezeichnet werden kann (Büchter und Gramlinger 2005). Nicht zuletzt aufgrund der Arbeitsmarktreformen und der Einführung des Systems der Bildungsgutscheine waren die Anbieter 
von beruflicher Weiterbildung in den letzten Jahren einem verstärkten Wettbewerbsdruck ausgesetzt, der zu einem Prozess des Strukturwandels und der Konsolidierung geführt hat. Die von den AfA ausgegebenen Bildungsgutscheine spezifizieren lediglich das Weiterbildungsziel, so dass sich die Kundinnen und Kunden selbst einen zertifizierten Anbieter auswählen können.

Der Markt der beruflichen Weiterbildung ist stark fragmentiert. Es gibt unterschiedliche Kundengruppen mit sehr unterschiedlichen Anforderungen und Größen. 2012 haben an den Weiterbildungskosten individuelle Teilnehmer 40\%, Betriebe $42 \%$, die Bundesagentur für Arbeit $5 \%$ und die weitere öffentliche Hand gut $13 \%$ getragen (Marcel 2015). Aufgrund der standardisierten Anforderungen und der relativ guten Planbarkeit ist der Markt für arbeitsmarktpolitische Weiterbildung für die meisten gewinnorientierten privaten Anbieter attraktiv. Auch Beschäftigte, die bW privat bezahlen, um ihren Arbeitsplatz zu sichern oder ihre Aussichten auf einen anderen Arbeitsplatz zu verbessern, sind an standardisierten Kursen mit weithin anerkannten Zertifikaten interessiert.

Unternehmen hingegen sind eher an betriebsspezifischen Angeboten interessiert, wie sich zum Beispiel auch in den Interviews von Brink et al. (2011) mit kleinen und mittleren Betrieben einer Region zeigt. Diese Betriebe wünschen sich „Praxisnähe, maßgeschneiderte Inhalte und zeitliche Flexibilität“. Sie finanzieren eher Anpassungsweiterbildungen, die nicht zu zertifizierten Abschlüssen führen und oft gesetzlich vorgeschrieben sind (Bläsche et al. 2017). Brink et al. (2011, S. 51) haben in der von ihnen untersuchten Fallregion beobachtet, dass ,dem Bedarf der Unternehmen nach kurzfristigen, zeitlich und inhaltlich flexiblen Weiterbildungsmaßnahmen ... nur sehr bedingt ein passendes Angebot gegenüber“" steht.

\subsection{Netzwerke in der bW}

Mit der Stärkung von Marktmechanismen in der Koordination der bW ging ein neues Verständnis der Rolle des Staates einher, dem nun „die Rolle eines aktivierenden, koordinierenden und stützenden Akteurs“"(Gnahs 2010, S. 327) zugeschrieben wird. Dadurch gewinnen Steuerungsformen zwischen Markt und Hierarchie an Bedeutung (Hartz und Schrader 2008, S. 17), die oft durch das Governance-Konzept beschrieben werden (Dingeldey et al. 2015), in dem Netzwerke eine herausragende Rolle spielen. Mit Blick auf Dienstleistungsmärkte schreibt Gummesson (2006), dass Interaktionsmuster zwischen Anbietern und Kunden eher eine Netzwerk- oder eine ,many-tomany"-Form als eine dyadische, also nur zweiseitige Beziehung, annehmen sollen. Netzwerke bezeichnen primär spontan entstehende und sich autonom stabilisierende Interaktionsmuster. Der Versuch, Netzwerke als Instrumente horizontaler Steuerung durch Politik und Verwaltung oder andere Akteure zu installieren, erzeugt einen gewissen inneren Widerspruch und oft auch Widerstand (Jütte 2002). Akteure in zentralen Netzwerkpositionen können hingegen durchaus als (Informations-)Broker oder Intermediäre koordinierend zwischen anderen Netzwerkbeteiligten agieren.

Viele Studien und vor allem auch politische Konzepte im Bereich der bW der letzten Jahre betonen vor diesem Hintergrund die Bedeutung von Kooperationen und Netzwerken (Brink et al. 2011). Tietgens (1991, S. 102) erläutert anschaulich: „Arbeitsvoraussetzungen müssen immer neu recherchiert, Meinungsführer müssen 
angesprochen, Kursleiter gewonnen, Räume ausgehandelt, Teilnehmer gehalten werden." Betont werden vor allem die positiven Seiten von Netzwerken (Diettrich 2014); mögliche nachteilige Effekte zum Beispiel auf den Wettbewerbsmechanismus des Marktes werden selten thematisiert.

Die Schwierigkeiten, funktionierende Kooperationen zu etablieren und vor allem aufrecht zu erhalten, werden aber zunehmend erkannt (Schiersmann 2014). Die Pflege von Netzwerken ist transaktionskostenintensiv. Netzwerke sind in der Regel klein genug, damit die Entscheidungen einzelner Akteure einen spürbaren Einfluss auf das gesamte Netzwerk haben. Aus diesem Grund erfordern und erzeugen funktionierende Netzwerke Vertrauen, das sich meist erst über längere Zeiträume hinweg aufbaut (Granovetter 2005). Dementsprechend sind Netzwerke deutlich schwerer als Märkte und Hierarchien durch administrative oder politische Vorgaben zu beeinflussen, siekönnen die Regulation je nach bestehenden Anreizen unterstützen oder unterlaufen (Rhodes 1996). Hertel (2013) zeigt, dass die zentrale Herausforderung weitergehender Kooperationen darin besteht, die verschiedenen Funktionslogiken der beteiligten Parteien aus Verwaltung, Privatwirtschaft sowie anderen Einrichtungen der Wirtschaft und des Bildungsbereichs zu integrieren und auf das gemeinsame Ziel auszurichten.

Callan und Ashworth (2004) zeigen für den australischen Fall unter anderem, dass eine Zusammenarbeit in Partnerschaften von Branchen und ihren Unternehmen auf der einen und Anbietern beruflicher Weiterbildung auf der anderen Seite oft durch geographische Nähe begünstigt und als wirtschaftliche Beziehung mit klaren formalen Regelungen organisiert wird. Brink et al. (2011) hingegen zeigen für Deutschland, dass Unternehmen sich in informellen Netzwerken mit heterarchischen Strukturen organisieren, um eine kritische Masse für die Nachfrage nach spezifischen Weiterbildungsangeboten zusammen zu bekommen.

\section{Analyse von regionalen Systemen der beruflichen Weiterbildung}

Die vorliegende Studie untersucht das Ineinandergreifen der verschiedenen Koordinationssysteme im Bereich der bW auf regionaler Ebene und borgt sich dazu von der Industrieökonomik das heuristische „,Structure-Conduct-Performance“-Konzept. Das „Structure-Conduct-Performance“-Konzept wird in der Industrieökonomik zur Beschreibung des Zusammenhangs zwischen Marktstruktur, Marktstrategien und Leistung bzw. Wettbewerbsfähigkeit der Marktteilnehmer herangezogen (Bain 1968). Es hilft dabei, die Struktur des Koordinationssystems, die Strategien der unterschiedlichen beteiligten Organisationen und die sich daraus ergebende Leistungsfähigkeit von Anbietern und Systemen in einem Kontext zu analysieren (Abb. 1).

Mit Blick auf Strategien und Leistung befinden sich viele Bildungsanbieter angesichts der hohen Diversität auf der Nachfrageseite in einem ,diversity-efficiency dilemma“ (Waslander 2007), und damit vor der Frage, wo zwischen teurer Anpassung an Kundenbedürfnisse und kostensparender Standardisierung des Angebots die für sie optimale Strategie liegt. Deshalb werden in der Analyse die Strategien der Standardisierung, Modularisierung und Kundenanpassung (,,customization“) herangezogen, um die Strategien der Anbieter, aber, soweit möglich, auch der intermediären 


\section{Struktur}

Koordination von Bedarf und Angebot

Markt - Netzwerk - Hierarchie

Strategien

Handlungen von Anbietern und Brokern

Standardisierung - Modularisierung - Kundenanpassung

Leistung

Kapazität und Fähigkeit des Systems

Statische Qualität - Anpassungsfähigkeit - Gestaltungskraft

Abb. 1 Analyserahmen (Quelle: Eigene Darstellung)

Organisationen der Systeme der bW im Rahmen des geborgten ,structure-conductperformance“ Konzeptes zu charakterisieren. Modularisierung kann als ,kundenindividuelle Massenproduktion“ („,mass customization“; Davis 1989), als Strategieoption zwischen den beiden Extremen der Standardisierung und Kundenanppassung gewertet werden (Sundbo 1994).

Die Leistung (,,performance“) kann aufgrund der Marktunvollkommenheiten und der nicht nur gewinnorientierten Anbieter und Intermediäre im Kontext der bW nicht (nur) über den Erfolg am Markt bzw. die Wettbewerbsfähigkeit definiert werden. Sie zeigt sich vor allem darin, inwiefern durch die Anbieter in verschiedenen Regionen die Bedarfe aller Kundengruppen, also der Beschäftigten, der Arbeitssuchenden, der Arbeitsverwaltung und der Unternehmen bedient werden. Die ,statische Qualität“ des lokalen Systems der bW beschreibt die Bedienung bestehender, expliziter Bedarfe. Die Nachfrage muss aber angesichts eines möglichen partiellen Marktversagens im Bereich der bW, mit ihren positiven externen Effekten und der Bedeutung einer Ko-Produktion des Wissens (s. Kap. 1) oft erst geschaffen werden. Auch der NichtTeilnehmer oder die Nicht-Teilnehmerin muss, anders ausgedrückt, als wichtiger Akteur im System der bW verstanden werden (Martin und Schömann 2015). Die „,dynamische Qualität“ des Systems beschreibt dementsprechend wie gut es gelingt, Teilnehmende für die bW zu mobilisieren, sich an ihre Bedarfe anzupassen und mit ihnen gemeinsam ein attraktives Angebot zu gestalten.

Struktur (,structure“) wird in der Industrieökonomik in der Regel quantitativ über die Konzentration des Marktes beschrieben. Britton et al. (1992) haben theoretisch und praktisch überprüft, in wie fern sich das Konzept auch im Dienstleistungsbereich anwenden lässt und sind u.a. auf das Problem fehlender Daten zur Erfassung der Marktstruktur gestoßen. In Bezug auf die lokalen Koordinationsmechanismen zur bW wird der Anwendungsbereich hier auf einen Dienstleistungsfall erweitert, in dem staatliche und quasi-staatliche Organisationen und Institutionen eine große Rolle 
spielen. Aus dieser Erweiterung ergibt sich, dass die Struktur anders als im reinen Marktfall nur qualitativ-beschreibend erfasst werden kann (s. Kap. 2).

\subsection{Fallstudien und Fallstudienergebnisse}

Mithilfe des beschriebenen Analyserahmens wurden im Rahmen komparativer explorativer Fallstudien regionale Systeme der bW analysiert. Für die Untersuchung wurden drei verschiedene Fallregionen in Niedersachsen ausgewählt. ${ }^{1}$ Dabei handelt es sich um eine städtische Arbeitsmarktregion (StA) mit einem Oberzentrum und seinem Umland ${ }^{2}$ und um zwei „dünn besiedelte ländliche Arbeitsmarktregionen“ (LA1 und LA2). Die administrativen Grenzen hatten für die Analyse keine weitere Bedeutung, sie definierten lediglich den jeweiligen geographischen Ausgangspunkt. Die befragten Beteiligten der regionalen Systeme der bW wurden im Schneeballsystem im Zuge der Befragung selbst ermittelt und definierten die Region der Aktivität ihrer Organisation jeweils selbst. Es zeigte sich im Zuge der Interviews und ihrer Auswertung, dass eine Unterscheidung nach Oberzentrum (StAO) und seinem Umland (StAU) sinnvoll war, da gravierende strukturelle Unterschiede im System der bW zu beobachten waren. Es wird also im Folgenden zwischen vier Regionen unterschieden.

Durch Vorab-Recherchen und durch Erfragen bei den jeweiligen Interviewpartnern wurden die wichtigsten Organisationen im Bereich der bW jeder Fallregion identifiziert. Darunter waren in allen Regionen die lokale Geschäftsstelle der AfA und das lokale Jobcenter, wobei allerdings die Vertreter des Jobcenters in LA2 nicht für ein Interview zur Verfügung standen (Abb. 2).

Die Jobcenter in StAU und in LA2 werden in kommunaler Verantwortung von sogenannten Optionskommunen betrieben, die anderen beiden Jobcenter befinden sich im Geschäftsbereich der jeweiligen AfA. Außerdem befragt wurden Vertreter der Industrie- und Handelskammern (IHK), der Handwerkskammern (HWK), der Volkshochschulen (VHS) sowie ausgewählter Privatanbieter. Bei den Vertretern der

\begin{tabular}{|c|c|c|c|c|c|c|c|c|}
\hline \multirow[b]{2}{*}{ Fallregionen } & \multicolumn{8}{|c|}{ Interviewpartner und Anzahl der Interviews } \\
\hline & & AfA & $\begin{array}{l}\text { Jobcenter } \\
\text { kommunal }\end{array}$ & $\begin{array}{c}\text { Jobcenter } \\
\text { mit Afa }\end{array}$ & HWK & IHK & VHS & $\begin{array}{c}\text { Privat- } \\
\text { anbieter }\end{array}$ \\
\hline $\begin{array}{l}\text { Städtische Arbeitsmarktregion, } \\
\text { Oberzentrum }\end{array}$ & StAO & 1 & & 1 & 1 & 1 & 1 & \\
\hline $\begin{array}{l}\text { Städtische Arbeitsmarktregion, } \\
\text { Umland }\end{array}$ & StAU & 1 & 1 & & & & 1 & 3 \\
\hline $\begin{array}{l}\text { Dünn besiedelte ländliche } \\
\text { Arbeitsmarktregion } 2\end{array}$ & LA2 & 1 & 0 & & 1 & 1 & 1 & \\
\hline $\begin{array}{l}\text { Dünn besiedelte ländliche } \\
\text { Arbeitsmarktregion } 1\end{array}$ & LA1 & 1 & & 1 & & 1 & 1 & 1 \\
\hline
\end{tabular}

Abb. 2 Erhebungsdesign in den Fallregionen (Quelle: Eigene Darstellung)

\footnotetext{
1 Die Regionen werden aufgrund des Datenschutzes nicht benannt, da wegen der geringen Anzahl größerer Anbieter von bW in einigen der Regionen sonst die Anonymität der Befragten nicht gewährt wäre.

2 Die Charakterisierung erfolgt nach den „Siedlungsstrukturellen Arbeitsmarktregionstypen 2014“ des Bundesinstituts für Bau-, Stadt- und Raumforschung (BBSR).
} 
HWK handelte es sich um einen Koordinator für Weiterbildung in der für LA1 und LA2 zuständigen HWK, sowie um einen leitenden Mitarbeiter eines Berufsbildungs- und Technologiezentrums (BTZ) in StAO. Es gab in den vier Regionen drei zuständige IHK, für die Analyseregionen StAO und StAU ist dieselbe IHK zuständig. In jeder Analyseregion konnte jeweils ein Vertreter oder eine Vertreterin einer VHS befragt werden. Die vier befragten großen Bildungsanbieter verfügen alle über mehrere Standorte. Nur in einem Fall (Firm 4) wurde ein Vertreter einer kleineren Geschäftsstelle in einer speziellen Analyseregion befragt, in allen anderen Fällen waren die Gesprächspartner Personen mit zentralen Aufgaben. Drei der vier großen befragten Anbieter haben Geschäftsstellen im Oberzentrum der städtischen Fallregion (StAO), jeweils einer von ihnen hat außerdem auch eine in jeweils einer der zwei ländlichen Analyseregionen (LA1 und LA2).

Die Gespräche wurden als halboffene leitfadenstrukturierte Interviews durchgeführt. Die Interviewleitfäden unterschieden sich vor allem nach den Gruppen Arbeitsverwaltung, Kammern und sonstige Anbieter einschließlich VHS. In allen Interviews wurde nach der internen Struktur („Hierarchie“) der Organisation, nach einer Beurteilung der lokalen Weiterbildungsmärkte, nach den lokalen Netzwerken im Bereich der bW und nach den Planungsprozessen in der Organisation gefragt. Außer in der Arbeitsverwaltung wurden außerdem Fragen zu der jeweiligen Marktstrategie der Organisation, den Aktivitäten im Bereich der Entwicklung von Angeboten und dem spezifischen Angebot im Bereich der bW gestellt.

Die Interviews wurden transkribiert und mithilfe der Software MaxQDA codiert. Die vergleichende Analyse auf der Ebene der Organisation und der Organisationstypen wurde durch Fallrekonstruktionen vorbereitet, wobei im ersten Schritt eine detaillierte Charakterisierung jeder Organisation in Bezug auf die benannten Themenfelder vorgenommen wurde. Im zweiten Schritt wurden ausgehend von den Eigenschaften der Organisationen und den Aussagen ihrer Vertreter sowie einiger ergänzender Informationen die Regionen nach Marktsituation und Netzwerkstrukturen sowie der Leistung des lokalen Systems der bW charakterisiert. In der abschlieBenden vergleichenden Analyse wurden mögliche Zusammenhänge zwischen der regionalen Struktur, der Struktur und Strategie der Anbieter und der Leistung des Systems hergestellt. Zunächst wird anhand der Interviews die Koordinationsstruktur in Hierarchien, Märkten und Netzwerken in den vier regionalen Weiterbildungssystemen rekonstruiert. Es folgt die Beschreibung der beobachteten Strategien und die Beurteilung der Leistungen der Systeme. Die Beobachtungen zur Struktur werden entlang der Dimensionen Hierarchie, Markt und Netzwerk diskutiert.

\subsection{Ergebnisse zur organisationsinternen hierarchischen Steuerung}

Abgeleitet aus Abschn. 2.1 werden der Beurteilung der beobachteten Hierarchien die Dimensionen Steuerung vs. Handlungsspielraum zugrunde gelegt. Stärkere, zentralisiertere interne Steuerung vergrößert bis zu einer gewissen Grenze tendenziell durch die Realisierung positiver Skaleneffekte die Effizienz der Organisationen. Mehr Handlungsspielräume auf der operativen Ebene der Organisation hingegen erhöhen im Idealfall ihre Anpassungsfähigkeit und so tendenziell die Effektivität von Aktivitäten. 
Die in Jobcentern und AfA geführten Interviews bestätigten im Hinblick auf die administrativen Steuerungsprozesse weitgehend die in der Literatur bereits beschriebenen Entwicklungen hin zu einer stärkeren Zielsteuerung und größerem individuellen Entscheidungsspielraum auf allen Ebenen der Hierarchie im Rahmen des Paradigmas zum New-Public-Management (s. Abschn. 2.1). Die aufgrund der entspannten Arbeitsmarktlage im Beobachtungszeitraum relativ günstige finanzielle Situation der Arbeitsagenturen hat die Stärkung der Gewichtung von Effektivität gegenüber Effizienz weiter begünstigt. Obwohl den einzelnen Mitarbeitenden so zum Beispiel eine verstärkte Beteiligung an regionalen Netzwerken ermöglicht wurde, wirkt sich vor allem der begrenzte Zugang zu Informationen zu den Bedarfen in den Betrieben vor Ort limitierend auf ihre Handlungsmöglichkeiten aus.

$I H K$ und $H W K$ sind innerhalb der größeren Kammerbezirke lokal durch Geschäftsstellen, Kreishandwerkerschaften und Innungen vertreten. Aus den dezentralen Einheiten gehen Informationen über die Bedarfe der Betriebe in den Regionen an die Kammern. Dabei geht es allerdings vor allem um Fragen der Ausbildung, weil die Betriebe selten Bedarfe im Bereich der Weiterbildung äußern.

Die vier befragten $V H S$ befinden sich in kommunaler Trägerschaft bzw. in Trägerschaft durch kommunale Zweckverbände und werden zusätzlich im Rahmen des niedersächsischen Erwachsenenbildungsgesetzes (NEBG) gefördert. Die unterschiedliche finanzielle Unterstützung aus den Kommunen wirkt sich auf die Ressourcenausstattung und die Handlungsmöglichkeiten der VHS aus, die in der städtischen Region mit StAO und StAU deutlich besser sind als in den ländlicheren Regionen.

Alle vier privaten Anbieter sind mit relativ selbständigen Geschäftsstellen, die sich weitgehend selbst tragen müssen, organisiert, wobei es immer ein zentrales Weiterbildungsangebot gibt. Von der Führung in der Zentrale, die auch zentrale Dienstleistungen anbietet, werden Empfehlungen ausgesprochen oder bestimmte Unterstützungsangebote gemacht. Manchmal gibt es zwischen Zentrale und Geschäftsstellen eine Zwischenebene, die den Austausch benachbarter Standorte koordiniert. Operative Entscheidungen werden in der Regel vor Ort in den Geschäftsstellen getroffen, während Entscheidungen mit strategischer Bedeutung mit der Geschäftsführung in der Zentrale abgestimmt werden.

Angebote für Privatkunden und Firmenkunden müssen nicht zertifiziert werden und können deshalb bei privaten Anbietern, IHK, HWK und VHS gleichermaßen vor Ort selbständig entwickelt werden. Außerdem werden Angebote im Zuge der Umsetzung bedarfsgerecht angepasst. Auch zu zertifizierende kleinere Kurse können in der Regel vor Ort entwickelt werden, wenn sich entsprechende Bedarfe abzeichnen. In der Entwicklung wird oft auf bestehende Module zurückgegriffen, sonst müssen, wenn es um den Erwerb von Zertifizierungen und Abschlüssen geht, Qualitätskriterien des Qualitätsmanagements beachtet werden. Die Zentrale von zwei der privaten Unternehmen ist in dieser Hinsicht eher restriktiv, indem von Anfang an nur Entwicklungen mit hohen Erfolgschancen zur Zertifizierung zugelassen werden, während Firm 4 in LA1 in dieser Hinsicht in seinem Unternehmen alle Freiheiten genießt und sich dementsprechend in Reaktion auf die lokalen Bedingungen in seinen Geschäftsfeldern auch deutlich anders entwickelt hat als etwa der Standort der Zentrale. 


\subsection{Ergebnisse zu regionalen Märkten für bW}

Abgeleitet aus Abschn. 2.2 und 2.3 werden der Beurteilung der beobachteten Märkte und Netzwerke die Dimensionen Konkurrenz vs. Kooperation zugrunde gelegt. Konkurrenz erhöht tendenziell die Effizienz der Organisationen und Angebote. Kooperation verbessert im Fall partiellen Marktversagens potentiell die Effektivität von Aktivitäten durch bessere Innformationen und Anpassungsmöglichkeiten.

Die Charakterisierung der Märkte aus Perspektive der am System der bW Beteiligten basiert auf den Einschätzungen der Befragten aus Arbeitsverwaltung und VHS, die sich jeweils genau einer Region zuordnen lassen, und die es in allen Regionen gibt, so dass eine gewisse Vergleichbarkeit der Einschätzungen gewährleistet ist. Die Netzwerkbeziehungen zwischen Arbeitsverwaltung, Anbietern, Wirtschaft und Kammern werden aus der Perspektive der VHS und der privaten Anbieter beschrieben.

Die Marktsituation ist in allen vier Regionen unterschiedlich: Das Oberzentrum StAO hat eine vielfältige Anbieterlandschaft mit starkem Wettbewerbsdruck; im Umland StAU selbst werden nicht mehr alle Bereiche der bW abgedeckt und es findet weiter ein Strukturwandel unter den Anbietern statt, das nahe Oberzentrum sorgt aber für ein gutes Angebot. Der Markt in LA2 ist aufgrund der geringen Bevölkerungsdichte und der geringen Arbeitslosigkeit unattraktiv für Anbieter, so dass es vor Ort nur wenige Angebote gibt und das virtuelle Klassenzimmer eine wichtige Alternative darstellt.

In der peripheren Region LA1 gibt es wenige etablierte Anbieter, die den Markt unter sich aufgeteilt haben. Um einen ruinösen Wettbewerb zu verhindern, kommt es hier unter den etablierten komplementären Anbietern zu einer kooperativen Koordination im Sinne der Idee der „Co-opetition“ (Brandenburger und Nalebuff 1996). Dementsprechend berichtet Firm 4 hier gleichzeitig von Kooperation und von starker Konkurrenz unter den Anbietern. Unterstützt wird die Stabilisierung des existierenden Angebots durch eine entsprechende Auftragspolitik der öffentlichen Kunden. Die Koordination jenseits der Märkte erschwert allerdings auch den Marktzugang für zusätzliche Anbieter.

\subsection{Ergebnisse zu regionalen Netzwerken für bW}

Im Vergleich zu den anderen Fallregionen ist LA1 aufgrund ihrer speziellen MarktSituation eine Region mit relativ intensiven Netzwerkbeziehungen. Der private Anbieter Firm 4 in LA1 hat einen engen Kontakt zur Arbeitsverwaltung und ist eine der wenigen Organisationen, die von engeren Kontakten zu den Kammern berichten. Die VHS in LA1 ist zudem die einzige VHS mit intensiven Beziehungen zur AfA. Ein enger Kontakt zwischen der VHS und dem Jobcenter besteht vor allem in StAU, wo sich das Jobcenter in kommunaler Trägerschaft befindet.

Es gibt Versuche, zum Beispiel von Seiten der Wirtschaftsförderungen, regionale Weiterbildungsnetzwerke für den regelmäßigen Austausch zu etablieren. Diese Netzwerke sind aber, wie die Interviews zeigen, für das operative Geschäft nur von schwacher Bedeutung. Die Beziehungen zu den anderen Anbietern sind überall stark von Anreizen des Marktes bestimmt. Kooperationen werden eingegangen, 
wo es durch Ausschreibungen vorgeschrieben ist, oder wenn Projektbeteiligungen Kompetenzen und Kapazitäten erfordern, die die eigene Organisation nicht besitzt. Firm 4 in LA1 ist aufgrund ihrer Spezialisierung auf Jobcenter-Kunden mit jeweils spezifischen Bedarfen in hohem Ausmaß auf Kooperationen angewiesen. Lediglich Firm 1 gibt explizit an, in derartigen Kooperationen eine Chance zur strategischen Weiterentwicklung zu sehen. Die Kammern sehen ihr Angebot als Ergänzung des Angebots privater Anbieter an. Sie werden von den anderen Anbietern aber entweder als Wettbewerber oder als notwendige Partner im Prüfungsbereich angesehen; eine darüberhinausgehende Kooperation gibt es nur selten.

Beziehungen zu den Arbeitgebern außerhalb der Weiterbildungsbranche und somit zu potentiellen Firmenkunden sind aus Sicht der VHS ebenso wie der privaten Anbieter schwierig zu etablieren. Intensivere Kontakte bestehen zu bestimmten Branchen, in denen die Anbieter spezialisiert sind, oder zu großen öffentlichen Arbeitgebern unter den Kunden. Ein wichtiger Weg, um den Kontakt herzustellen, sind für die privaten Anbieter Umschulungen, da die entsprechenden Schüler und Schülerinnen für den praktischen Teil der Ausbildung auf Plätze in den Unternehmen angewiesen sind. Auch die Förderung WeGebAU, die bestimmte Gruppen Beschäftigter in der bW fördert, öffnet manchmal den Zugang zu Betrieben.

Mehr Möglichkeiten zu Kontakten zu den Unternehmen vor Ort haben die Arbeitsverwaltung und die Kammern, für die sich Kontakte im Zusammenhang mit der Ausbildung und der Mitgliederpflege ergeben. Die AfA haben in den vergangenen Jahren im Zuge der Reformen, die auch den Handlungsspielraum der operativen Ebene vergrößern sollten (s. Abschn. 3.1), den Arbeitgeberservice stark ausgebaut. Der unmittelbare und intensive Austausch findet allerdings häufig anlassbezogen mit größeren Unternehmen statt. Das Interesse kleinerer Unternehmen zum Beispiel an der Qualifizierungsberatung ist begrenzt. Die Zusammenarbeit zwischen Kammern, anderen Arbeitgeberverbänden und Arbeitsverwaltung hängt stark vom Zuschnitt der regionalen Zuständigkeiten und vom jeweiligen Sitz der Einrichtungen ab. Aus Sicht der AfA in LA2 etwa ist insbesondere die HWK zu weit entfernt für einen engen Austausch.

\subsection{Ergebnisse zu Strategien und Leistungen}

Die Strategien der Anbieter werden im Spannungsfeld zwischen Diversität und Effizienz danach beurteilt, ob sie standardisiert, modular oder kundenangepasst sind (s. oben). Bask et al. (2011) unterscheiden für Dienstleistungsanbieter zwischen vier Extremtypen in der Kombination von Modularisierung und Kundenanpassung (,,customization"): nicht modular, standardisiert (1), modular standardisiert (2), modular, kundenangepasst (3) und nicht modular, kundenangepasst (4).

Standardisierte Angebote stehen im Zeichen der Effizienz und können eine hohe statische Qualität (s. Kap. 3) liefern. Aufstiegsweiterbildungen, die vor allem das Angebot der Kammern dominieren, sind stark standardisiert. Andere Indikatoren für statische Qualität sind die Vielzahl und Tragweite zertifizierter Angebote, die Existenz und Ausgestaltung von Qualitätssicherungssystemen sowie die Qualität des Lehrkörpers. 
Kundenangepasste Angebote stehen im Zeichen der Effektivität und der dynamischen Qualität. Ein Maß für das Ausmaß der Kundenanpassung ist die Kundenbeteiligung an der Erzeugung der Dienstleistung (Bask et al. 2011). Bei Firmenschulungen ist die Möglichkeit zur Anpassung an Kundenwünsche allgemein hoch, denn Unternehmen legen oft wenig Wert auf zertifizierte Abschlüsse. Ihre relative Bedeutung für einen Anbieter kann daher als Gradmesser der dynamischen Qualität von Angeboten gesehen werden. Dynamische Qualität äußert sich außerdem in einer Ko-Produktion von Wissen und Mobilisierung immer neuer (Firmen-)Kunden.

Modulare Strategien bieten einen Mittelweg für die Balance zwischen Effizienz und Effektivität bzw. zwischen statischer und dynamischer Qualität. Ein Maß für das Ausmaß der Modularisierung ist die Anzahl der in verschiedenen kombinierbaren Modulen angebotenen Varianten (Bask et al. 2011). Zu den Managementanforderungen der modularen Kundenanpassung gehört, dass Wissen geteilt und neue Kundenbedürfnisse ständig in neue Dienstleistungen übersetzt werden müssen. Erforderlich sind also eine offene Wissenskultur und die In-house-Entwicklung neuer Produkte und Prozesse (Da Silveira et al. 2001).

\subsubsection{Statische Qualität der Anbieter}

Die statische Qualität der Angebote ist gemessen an der Vielfalt der angebotenen Zertifikate insgesamt bei den privaten Anbietern deutlich höher als bei den VHS. Die großen Anbieter können die Overheadkosten aus Planung und Entwicklung auf viele Standorte verteilen und bei entsprechend dezentraler Organisation und funktionierenden Kommunikationsstrukturen im Unternehmen von den Erfahrungen an allen Standorten lernen. Diese Strukturen kombiniert mit einem hohen Wettbewerbsdruck haben die Entwicklung neuer modularer und digitaler Angebotsformen begünstigt. Online-Angebote, die günstige standortunabhängige Angebote und flexibles Lernen ermöglichen können, spielen bei den Kammern und in den VHS noch eine geringe Rolle, sind aber für die privaten Anbieter Firm 2 und Firm 3 von hoher Bedeutung. Nur die entsprechende Infrastruktur ermöglicht es Firm 3 in Region LA2, in der es sonst kaum Anbieter gibt, ein breites Angebot vorzuhalten.

Unter dem hohen Wettbewerbsdruck spielt die Qualitätssicherung vor allem bei den privaten Anbietern eine große Rolle. Bei ihnen gibt es Selbstevaluierungsroutinen, regelmäßige Teilnehmendenbefragungen, automatische Erfolgskontrollen sowie Audits der verschiedenen Zertifizierer. Über allem steht bei den privaten Anbietern mit ihrer Ausrichtung auf die Kunden der Rechtskreise SGBII und SGBIII die Vermittlungsquote als Erfolgskennzahl. Die Kammern arbeiten im Bereich der Qualitätssicherung vor allem mit dem direkten Feedback ihrer Mitglieder und mit Evaluationsbögen für Teilnehmende an Weiterbildungen. In den VHS sind Teilnehmerbefragungen wichtigster Bestandteil des Qualitätssicherungssystems.

Die Qualität des Lehrkörpers kann hier nur mit Blick auf die Qualität der Beschäftigungsverhältnisse und die sich daraus ergebende Attraktivität der Stellen beurteilt werden. In Firm 1 ist der Anteil der festangestellten Dozentinnen und Dozenten in den letzten Jahren auf $95 \%$ gestiegen, weil das als Basis eines verlässlichen und flexiblen Angebots gesehen wird. Bei allen privaten Anbietern liegt der Anteil zwischen $40 \%$ und $95 \%$ und somit deutlich höher als bei Kammern und VHS. Insbesondere in 
LA2 mit dem sowieso geringen Angebot an bW fällt es der VHS auch aufgrund der relativ unattraktiven Beschäftigungsverhältnisse schwer, die benötigten Lehrkräfte zu finden. Die Kammern wollen durch die nebenberufliche Lehrtätigkeit Praxisnähe erreichen, doch auch dort gibt es freiberufliche Dozenten und Dozentinnen im Hauptberuf.

\subsubsection{Dynamische Qualität der Anbieter}

Die Kammern hätten aufgrund ihrer relativ guten Kontakte zu den Arbeitgebern (s. Abschn. 3.4) ein Potential für die Entwicklung kundenangepasster Angebote. Sie sind aber eher auf die abschlussorientierten und damit standardisierten Bereiche der Aus- und Weiterbildung ausgerichtet. Nur IHK 1 in der städtischen Fallregion berichtet von einem etwas höheren Anteil an Inhouse-Schulungen und davon ausgehenden Impulsen für die Angebotsentwicklung. IHK 2 in LA1 unterstützt die Bündelung der Bedarfe von Unternehmen, indem aktiv auf bestimmte neue Themen aufmerksam gemacht wird. Das Angebot der Kammern selbst lässt sich am ehesten als „,modular standardisiert“" nach Bask et al. (2011; s. Abschn. 3.5) beschreiben.

In den städtischen Regionen StAO und StAU gehen die privaten Anbieter ebenso wie die VHS intensiv auf spezifische Wünsche der relativ wenigen Firmen unter ihren Kunden ein. Der Firmenkundenmarkt ist für sie ein von den Möglichkeiten der Preisgestaltung her attraktives Marktsegment. In den ländlichen Regionen steht ein Angebot der VHS für Firmen erst am Anfang und wird eher programmbezogen entwickelt. Die VHS agieren eher ,nicht modular, standardisiert“. Firm 2 und Firm 3 hingegen arbeiten „modular, kundenangepasst“; sie haben ein stark modular aufgebautes Angebot, das die starre Programmplanung durch eine höchst flexible kundenspezifische Auswahl ersetzt. Auch Firm 1, auf die das nicht im gleichen Ausmaß zutrifft, bemüht sich um eine entsprechende Entwicklung. Trotz der Bemühungen bleibt der Firmenkundenmarkt klein und die Mobilisierung neuer Firmenkunden gelingt nur selten. Große Arbeitgeber nutzen oft interne Lösungen, oft auch in Zusammenarbeit mit überregionalen Anbietern, während es vielen kleinen Firmen an Management- und Personalressourcen für einen systematischen Einsatz von bW mangelt.

Die interviewten privaten Anbieter richten sich stark auf die große Kundengruppe der staatlich geförderten Arbeitssuchenden aus. Sie stellen sich auf die Bedarfe der Arbeitsverwaltung, von der die Entwicklung des wichtigsten Marktsegmentes abhängt, flexibel ein. Angesichts der kritischen Masse an Kursteilnehmenden besteht hier allgemein die Möglichkeit, das Programm auch auf eine spontan auftretende Nachfrage hin zu ändern. Die Strategie von Firm 4 in LA1 mit der Ausrichtung auf durch das Jobcenter geförderte Kundengruppe lässt sich sogar als „,nicht modular, kundenangepasst" beschreiben.

\subsubsection{Leistungen der regionalen Systeme der $b W$}

Die Leistung regionaler Weiterbildungslandschaften insgesamt hängt neben der Leistung der einzelnen Organisationen davon ab, wie deren Angebote aufeinander abgestimmt sind und wie sie sich ergänzen. Hierbei kommt Netzwerkbrokern eine 
potentiell bedeutende Rolle zu. Die AfA haben durch die Bildungszielplanung und die Definition von Standards einen hohen Einfluss auf die Qualität eines großen Angebotssegments. Sie bemühen sich durch aktive Beteiligung an den Netzwerken der Regionen und den Kontakt zu Unternehmen unter anderem über den Arbeitgeberservice um Anpassung ihrer Zielplanungen an die Unternehmensbedarfe, indem sie zum Beispiel auch vorausschauend geplante größere Firmenniederlassungen am Standort berücksichtigen. Gleichzeitig können die AfA aber gerade in Zeiten niedriger Arbeitslosigkeit Qualifikationsbedarfe mit ihren Kunden oft nur begrenzt erfüllen. Aufgrund dieser Restriktionen und fehlender Kontakte zu einem Großteil der KMU erfasst die Bildungszielplanung nur einen Teilbereich des Bedarfs an bW.

Die Kehrseite der Bedeutung des Marktes für arbeitsmarktpolitische Weiterbildung ist, dass private Anbieter außerhalb der städtischen Zentren vor allem dort tätig sind, wo eine entsprechende Arbeitslosigkeit ein ausreichendes Kundenpotential verspricht. Insbesondere in peripheren Regionen wie LA2 mit wenigen Arbeitslosen und ohne eigene private Weiterbildungsanbieter ermöglichen zwar die neuen digitalen und modularen Angebotsformen ein größeres Angebot; sie erfordern allerdings wenig Personal vor Ort, was auch bedeutet, dass eine Anpassung an die dortigen Bedingungen über die modularen Gestaltungsmöglichkeiten hinaus kaum möglich ist.

Angebote von Kammern und VHS könnten das Fehlen privater Anbieter theoretisch teilweise oder ganz kompensieren. Das Angebot der Kammern ist aber auf die jeweiligen Kammerstandorte konzentriert, solange nicht eine Koordination der örtlichen Nachfrage von Unternehmen zum Beispiel durch die Innungen gelingt. Eine Broker-Funktion zwischen Unternehmen und anderen Anbietern nehmen die Kammern nicht ein. Die VHS gibt es in allen Gemeinden, sie haben aber gerade in den ländlichen Kreisen mit oftmals geringer kommunaler Finanzierung und unattraktiven Marktbedingungen nicht die Möglichkeit, die fehlenden Angebote anderer Anbieter zu kompensieren.

In LA1 verhinderte Netzwerkkoordination einen ruinösen Wettbewerbs unter den verbliebenen Anbietern (s. Abschn. 3.3). Es kann sein, dass diese Abschwächung des Wettbewerbs die statische Qualität des Angebots kurzfristig verringert, langfristig aber erhöht, weil ein ruinöser Wettbewerb verhindert wird. Der eingeschränkte Wettbewerb lässt außerdem Spielraum, um sich auf spezifische Anforderungen vor Ort einzustellen.

\section{Zusammenfassung und Schlussfolgerungen}

Es konnte gezeigt werden, dass sich eine unterschiedliche Nachfragedichte in verschiedenen Regionen weiter auf Management- und Netzwerkstrukturen und dann auch auf Angebotsformen und -qualitäten im Bereich der bW auswirkt. Insgesamt stehen angesichts der deutlichen Marktausrichtung des Systems bW Konkurrenz und Effizienz stärker im Vordergrund als Kooperation und Effektivität. Langfristig stabile Netzwerkbeziehungen zwischen verschiedenen Organisationen sind selten. Kooperationen werden eingegangen, wenn dadurch der Wettbewerbsdruck verringert oder wenn spezifischen Forderungen institutioneller Kunden entgegengekommen werden 
kann. In peripheren Regionen hat Netzwerkkoordination das Potential, auf Kosten von Marktsteuerung und Effizienz zur Stabilisierung eines an die lokalen Bedarfe angepassten Angebots beizutragen.

Vor allem die privaten Anbieter schaffen es, durch zentrale Entwicklung eine hohe Anzahl der von der Arbeitsverwaltung geforderten zertifizierten Angebote bereitzuhalten, um im starken Wettbewerb schnell auf neue Bedarfe reagieren zu können. Deshalb ist die statische Qualität des Angebots gemessen an den hier berücksichtigten Kriterien insbesondere in den urbanen Regionen mit hoher Anbieterdichte hoch.

Zugunsten lokal angepasster Angebote wirken Organisationsstrukturen, die trotz zentralen Controllings viel Handlungsspielraum in den einzelnen Geschäftsstellen belassen und einen Informationsaustausch zwischen Zentralen und Niederlassungen befördern. Das ermöglicht es den Anbietern, sich mit nicht zertifizierten Angeboten insbesondere auf Firmenkunden einzustellen, die angesichts der möglichen freien Preisgestaltung ein interessantes aber kleines Marktsegment darstellen. Fehlende Erfolge in der Mobilisierung potentieller weiterer Firmenkunden und entsprechend ungenaue Kenntnisse ihrer Bedarfe bei Anbietern und Brokern wie der AfA beschränken die dynamische Qualität des Systems.

Der spezielle institutionenökonomische Rahmen der Analyse kann nicht ohne größere Anpassungen auf Situationen übertragen werden, in denen dem Markt eine deutlich geringere Rolle zukommt als zurzeit in der bW in Deutschland. In diesem Kontext aber hat er sich als fruchtbar erwiesen, weil erst die parallele Analyse von Strukturen, Strategien und Leistungen die Identifizierung der komplexen Zusammenhänge zwischen ihnen ermöglichte. Es wurde deutlich, dass Erfolg oder Misserfolg einer Koordinationsform immer erst im Kontext der anderen vorhandenen Koordinationsformen verstanden werden kann. So ist Netzwerkkoordination in hart umkämpften Märkten mit vielen Anbietern nur begrenzt möglich und setzt außerdem bestimmte Managementstrukturen innerhalb der Organisationen mit ausreichenden Handlungsspielräumen vor Ort voraus.

Auf der Basis der durch diese Zusammenschau generierten Ergebnisse zur Frage nach der Qualität regionaler Weiterbildungslandschaften ist die in den letzten Jahrzehnten zunehmend forcierte Marktorientierung des deutschen Weiterbildungssystems ambivalent zu beurteilen. Einerseits produziert das System auch aufgrund des funktionierenden Wettbewerbs vor allem in den urbanen Regionen, gemessen an den hier zugrunde gelegten Kriterien, eine relativ hohe statische Qualität. Andererseits findet die für wissensintensive Dienstleistungen eigentlich so charakteristische Ko-Produktion von Wissen durch Anbieter und Kunden nur sehr eingeschränkt statt, und gerade in Regionen mit dünnen Arbeitsmärkten gelingt es nicht, Nachfrage nach bW zu motivieren (relativ geringe dynamische Qualität). Eine stärkere Spezialisierung von Anbietern mit der Möglichkeit, vermehrt auf spezifische lokale Bedarfe einzugehen findet sich ausgerechnet in dem relativ unattraktiven ländlichen Markt, wo der Wettbewerbsmechanismus durch Netzwerkkoordination teilweise außer Kraft gesetzt wurde.

Die Realisierung des politischen Ziels, Arbeitgeber und Arbeitnehmer zu einer größeren Beteiligung an bW zu motivieren, setzt also möglicherweise Handlungsspielräume jenseits des Wettbewerbs voraus, die es ermöglichen, eine stärkere Inter- 
aktion der Organisationen der Weiterbildung mit Arbeitgebern und Arbeitnehmern aktiv zu forcieren. Wenn man den Konkurrenzdruck mit seinen positiven Auswirkungen auf bestimmte Aspekte der statischen Qualität nicht reduzieren möchte, können diese Handlungsspielräume nur durch zusätzliche finanzielle Ressourcen oder die Umlenkung existierender finanzieller Ressourcen im arbeitsmarktpolitischen System erreicht werden. Deren Verteilung müsste im Wettbewerbskontext an klar definierte Ziele wie etwa höhere Weiterbildungsbeteiligungen oder mehr bedarfsgerechte Angebote in peripheren Regionen geknüpft sein. Ein Weg, um in diesem Sinne etwas zu erreichen, wäre die, möglicherweise auch regional begrenzte, nachfrageseitige Subventionierung von Firmenkunden. Wenn es so gelänge, ein größeres Engagement von Unternehmen im Bereich der bW zu induzieren, würden auch periphere Märkte attraktiver für Anbieter, die dann vor Ort den Unternehmen attraktive Angebote näher bringen und sie an ihre Bedarfe anpassen könnten. Eine solche vorübergehende nachfrageseitige Förderung von betrieblicher oder betrieblich finanzierter bW in peripheren Regionen müsste nach einer vorab festgelegten Frist aber wieder abgebaut und in eine überwiegend unternehmensfinanzierte Lösung überführt werden, um die Gefahr von Fehlanreizen zu minimieren.

Die Fallstudienergebnisse können nur begrenzt verallgemeinert werden. Weitere Untersuchungen zur Leistungsfähigkeit unterschiedlicher regionaler Systeme der Aus- und Weiterbildung werden gerade vor dem Hintergrund knapper werdender Fachkräfte, eines sich beschleunigenden technologischen Wandels und der Befürchtung wachsender räumlicher Disparitäten dringend benötigt.

Danksagung Das Forschungsvorhaben wurde mit Mitteln des Bundesministeriums für Bildung und Forschung unter dem Förderkennzeichen 033RA04A gefördert. Die Verantwortung für den Inhalt dieser Veröffentlichung liegt bei den Autoren.

Open Access Dieser Artikel wird unter der Creative Commons Namensnennung 4.0 International Lizenz (http://creativecommons.org/licenses/by/4.0/deed.de) veröffentlicht, welche die Nutzung, Vervielfältigung, Bearbeitung, Verbreitung und Wiedergabe in jeglichem Medium und Format erlaubt, sofern Sie den/die ursprünglichen Autor(en) und die Quelle ordnungsgemäß nennen, einen Link zur Creative Commons Lizenz beifügen und angeben, ob Änderungen vorgenommen wurden.

\section{Literatur}

Bain, J. S. (1968). Industrial Organisation. New York: Wiley.

Bask, A., Lipponen, M., Rajahonka, M., \& Tinnilä, M. (2011). Framework for modularity and customization. Service perspective. Journal of Business \& Industrial Marketing, 5, 306-319.

Bläsche, A., Brandherm, R., Eckhardt, C., Käpplinger, B., Knuth, M., Kruppe, T., Kuhnhenne, M., \& Schütt, P. (2017). Qualitätsoffensive strukturierte Weiterbildung in Deutschland. Working Paper Forschungsförderung 025. Düsseldorf: Hans-Böckler-Stiftung.

Borrás, S., \& Edquist, C. (2015). Education, training and skills in innovation policy. Science and Public Policy, 42, 215-227.

Bosch, G. (2017). Different national skills systems. In J. Buchanan, D. Finegold, K. Mayhew, \& C. Warhurst (Hrsg.), Oxford handbook of skills and training (S. 424-443). Oxford: Oxford University Press.

Bosch, G., \& Charest, J. (2008). Vocational training and the labour market in liberal and coordinated economies. Industrial Relations Journal, 39, 428-447.

Brandenburger, A. M., \& Nalebuff, J. (1996). Co-opetition. New York: Currency Doubleday.

Brater, M. (1980). Die Aufgaben beruflicher Weiterbildung: Zur Konzeption einer "subjektorientierten" Weiterbildung. In A. Weymann (Hrsg.), Handbuch für die Soziologie der Weiterbildung (S. 66-101). Darmstadt: Luchterhand. 
Brink, S., Führmann, B., \& Schuhen, M. (2011). Kooperative Weiterbildungsnetzwerke von Unternehmen - Netzwerkstruktur und Problemlösungsbeitrag. Report, 34(4), 47-57.

Britton, L.C., Clark, T.A.R., \& Ball, D.F. (1992). Modify or extend? The application of the structure conduct performance approach to service industries. The Service Industries Journal, 12(1), 34-43.

Buchanan, J. (2006). From 'skill shortages' to decent work. The role of better skill ecosystems. Sydney: NSW Board of Vocational Education and Training.

Büchter, K., \& Gramlinger, F. (2005). The System of Continuing Education in German VET. bwp@, 7. http://www.bwpat.de/7eu/buechter_gramlinger_de_bwpat7.shtml. Zugegriffen: 12.02.2018

Callan, V., \& Ashworth, P. (2004). Working together. Industry and VET provider training partnerships. Adelaide: NCVER.

Da Silveira, G., Borenstein, D., \& Fogliatto, F. S. (2001). Mass customization. Literature review and research directions. International Journal of Production Economics, 72(1), 1-13.

Dauth, C. (2017). Regional discontinuities and the effectiveness of further training subsidies for low-skilled employees. Paper 7/2017. Nürnberg: IAB Discussion.

Davis, S. M. (1989). From future perfect. Mass customizing. Planning Review, 17(2), 16-21.

Diettrich, A. (2014). Kooperationsraum. Lernen in Kooperationen und Netzwerken. In W. Wittwer, A. Diettrich \& M. Walber (Hrsg.), Lernräume. Gestaltung von Lernumgebungen für Weiterbildung (S. 163-170). Wiesbaden: Springer VS.

Dingeldey, I., Holtrup, A., \& Warsewa, G. (2015). Einleitung. Wandel der Governance von Erwerbsarbeit. In I. Dingeldey, A. Holtrup \& G. Warsewa (Hrsg.), Wandel der Governance der Erwerbsarbeit (S. 1-19). Wiesbaden: Springer VS.

Doerr, A., \& Kruppe, T. (2012). Bildungsgutscheine und Zertifizierungen aus Sicht der Arbeitsverwaltung. Umfrageergebnisse aus der Bundesagentur für Arbeit. IAB-Forschungsbericht 6/2012. Nürnberg: Bundesagentur für Arbeit.

Finegold, D. (1999). Creating self-sustaining, high-skill ecosystems. Oxford Review of Economic Policy, 15(1), 60-81.

Finegold, D., \& Soskice, D. (1988). The failure of training in Britain. Analysis and prescription. Oxford Review of Economic Policy, 4(3), 21-53.

Gnahs, D. (2010). Steuerung kommunaler Bildungslandschaften. In K. Birkelbach, A. Bolder \& K. Düsseldorff (Hrsg.), Berufliche Bildung in Zeiten des Wandels. Festschrift für Rolf Dobischat zum 60. Geburtstag (S. 323-337). Baltmannsweiler: Schneider Verlag Hohengehren.

Granovetter, M. (2005). The impact of social structure on economic outcomes. Journal of Economic Perspectives, 19(1), 33-50.

Grotlüschen, A., Haberzerth, E., \& Krug, P. (2010). Rechtliche Grundlagen der Weiterbildung. In R. Tippelt \& A. von Hippel (Hrsg.), Handbuch Erwachsenenbildung und Weiterbildung (3. Aufl. S. 347-366). Wiesbaden: Verlag für Sozialwissenschaften.

Gummesson, E. (2006). Many-to-many marketing as grand theory. A nordic school contribution. In R. F. Lusch \& S. K. Vargo (Hrsg.), The service-dominant logic of marketing: dialog, debate, and directions (S. 339-353). Armonk: ME Sharpe.

Hartz, S., \& Schrader, J. (2008). Steuerung und Organisation in der Weiterbildung. Ein vernachlässigtes Thema? In S. Hartz \& J.J. Schrader (Hrsg.), Steuerung und Organisation in der Weiterbildung (S. 9-30). Bad Heilbrunn: Klinkhardt.

Hertel, S. (2013). Interorganisationale Kooperation im Feld beruflicher Nachqualifizierung als Resultat struktureller Kopplung von Organisationen. Report, 36, 13-23.

Jütte, W. (2002). Soziales Netzwerk Weiterbildung. Analyse lokaler Institutionenlandschaften. Bielefeld: Bertelsmann.

Klingert, I., \& Lenhart, J. (2017). Jobcenter-Strategien zur Arbeitsmarktintegration von Langzeitarbeitslosen. IAB Forschungsbericht 3/2017. Nürnberg: Institut für Arbeitsmarkt- und Berufsforschung.

Lusch, R.F., \& Vargo, S.L. (2006). Service-dominant logic. Reactions, reflections and refinements. Marketing theory, 6(3), 281-288.

Maglio, P. P., \& Spohrer, J. (2008). Fundamentals of service science. Journal of the Academy of Marketing Science, 36(1), 18-20.

Marcel, W. (2015). Weiterbildungsfinanzierung in Deutschland: Gutachten für die Bertelsmannstiftung. Gütersloh: Bertelsmann.

Margarian, A., \& Lilje, A. (2014). Active employment policy in local labour market regimes. Conference Paper from the 7th Summer Conference in Regional Science in Marburg in June 2013.

Martin, A., \& Schömann, K. (2015). Zur Relevanz regionaler und kommunaler Akteurskonstellationen für das Weiterbildungsverhalten. In M. Schemmann (Hrsg.), Organisationsforschung in der Er- 
wachsenenbildung: Theorie, Methoden, Befunde. Internationales Jahrbuch der Erwachsenenbildung, Band 38. (S. 153-176). Wien, Köln, Weimar: Böhlau.

Martin, A., \& Schrader, J. (2016). Deutscher Weiterbildungsatlas. Kreise und kreisfreie Städte. Bonn: DIE.

Martin, A., Schömann, K., Schrader, J., \& Kuper, H. (2015). Deutscher Weiterbildungsatlas. Bielefeld: wbv.

Nuissl, E., \& Brandt, P. (2009). Porträt Weiterbildung Deutschland. Bielefeld: Bertelsmann.

Osborne, D., \& Plastrik, P. (1999). Banishing bureaucracy: The five strategies for reinventing government. San Francisco, CA: Jossey Bass.

Powell, W. W. (1990). Neither market nor hierarchy. Network forms of organization. Research in Organizational Behavior, 12, 295-336.

Regini, M. (1997). Different responses to common demands: firms, institutions, and training in Europe. European Sociological Review, 13(3), 267-282.

Rhodes, R. A.W. (1996). The new governance. Governing without government. Political Studies, 44(4), 652-667.

Robalino, D., Almeida, R., \& Behrman, J. (2012). Policy framework. The economic rationale for skills development policies. In R. Almeida, J. Behrman \& D. Robalino (Hrsg.), The right skills for the job? Rethinking training policies for workers (S. 49-66). Washington D.C: The World Bank.

Schiersmann, C. (2014). Hybridity and governance changes in continuing education. American Behavioral Scientist, 58(11), 1464-1473.

Schütz, H. (2008). Reform der Arbeitsvermittlung. Uniformierungsdruck in der Bundesagentur für Arbeit. Opladen, Farmington Hills: Budrich.

Sowa, F., \& Staples, R. (2014). Accounting in der Arbeitsverwaltung. Vermittlungsfachkräfte zwischen Steuerungsimperativen und autonomem Vermittlungshandeln. Zeitschrift für Sozialreform, 60(2), 149-173.

Sowa, F., Gottwald, M., Grimminger, S., Ixmeier, S., \& Promberger, M. (2016). Vermittlerhandeln im weiterentwickelten Zielsystem der Bundesagentur für Arbeit: Zum Forschungsdesign einer organisationsethnografischen Studie. IAB Forschungsbericht 2/2016. Nürnberg: IAB.

Sundbo, J. (1994). Modularization of service production and a thesis of convergence between service and manufacturing organizations. Scandinavian Journal of Management, 10(3), 245-266.

Tietgens, H. (1991). Einleitung in die Erwachsenenbildung (2. Aufl.). Darmstadt: Wissenschaftliche Buchgesellschaft.

Waslander, S. (2007). Mass customization in schools: strategies Dutch secondary schools pursue to cope with the diversity-efficiency dilemma. Journal of Education Policy, 22(4), 363-382.

Wegge, M. (1996). Qualifizierungsnetzwerke - Netze oder lose Fäden? Ansätze regionaler Organisation beruflicher Weiterbildung. Opladen: Leske + Budrich.

Zech, R. (2013). Identität von und Identifikation in Organisationen - am Beispiel von Weiterbildungsanbietern. Journal für Psychologie, 21(3). https://www.journal-fuer-psychologie.de/index.php/jfp/article/ view/302. Zugegriffen: 12.02.2018. 